\title{
Detailed Simulation of Complex Hydraulic Problems with Macroscopic and Mesoscopic Mathematical Methods
}

\author{
Chiara Biscarini, ${ }^{1}$ Silvia Di Francesco, ${ }^{2}$ Fernando Nardi, ${ }^{1}$ and Piergiorgio Manciola ${ }^{3}$ \\ ${ }^{1}$ Warredoc, University for Foreigners of Perugia, Via XIV Settembre, 06100 Perugia, Italy \\ ${ }^{2}$ CIPLA, Interuniversitary Centre for Environment, University of Perugia, Piazza Università 1, 06125 Perugia, Italy \\ ${ }^{3}$ DICA, Department of Civil and Environmental Engineering, University of Perugia, Via G. Duranti 93, 06125 Perugia, Italy
}

Correspondence should be addressed to Chiara Biscarini; chiara.biscarini@unistrapg.it

Received 30 January 2013; Accepted 15 March 2013

Academic Editor: Carlo Cattani

Copyright (c) 2013 Chiara Biscarini et al. This is an open access article distributed under the Creative Commons Attribution License, which permits unrestricted use, distribution, and reproduction in any medium, provided the original work is properly cited.

\begin{abstract}
The numerical simulation of fast-moving fronts originating from dam or levee breaches is a challenging task for small scale engineering projects. In this work, the use of fully three-dimensional Navier-Stokes (NS) equations and lattice Boltzmann method (LBM) is proposed for testing the validity of, respectively, macroscopic and mesoscopic mathematical models. Macroscopic simulations are performed employing an open-source computational fluid dynamics (CFD) code that solves the NS combined with the volume of fluid (VOF) multiphase method to represent free-surface flows. The mesoscopic model is a front-tracking experimental variant of the LBM. In the proposed LBM the air-gas interface is represented as a surface with zero thickness that handles the passage of the density field from the light to the dense phase and vice versa. A single set of LBM equations represents the liquid phase, while the free surface is characterized by an additional variable, the liquid volume fraction. Case studies show advantages and disadvantages of the proposed LBM and NS with specific regard to the computational efficiency and accuracy in dealing with the simulation of flows through complex geometries. In particular, the validation of the model application is developed by simulating the flow propagating through a synthetic urban setting and comparing results with analytical and experimental laboratory measurements.
\end{abstract}

\section{Introduction}

The detailed three-dimensional (3D) simulation of freesurface flows is a challenging task for engineering projects [1] dealing with open flow hydraulics at both the large and small scale because of the computational burden and the large parameterization needed for representing the physics, geometry, and boundary conditions. Nevertheless, in recent times, the always larger availability of remotely sensed data, providing digital topographic and land cover information, and the decreasing impact of the computational burden make the application of small-scale high-resolution 3D numerical models feasible and attractive for engineering studies. In particular, it is foreseen that 3D hydraulic simulations shall support not only fluid mechanics analyses for engineering design at the small scale, but also large-scale applications in urban planning, river restoration, and flood mitigation and management projects.

There are several recent works in the literature that report the ability of the Navier-Stokes (NS) method using the two-dimensional (2D) shallow water (SW) equations [2] to simulate river surface flows, even if the SW hypothesis is not always valid for the inertial effects and significant gradients [3-5]. In fact, several fully three-dimensional NS simulations are available in the literature for representing not only dambreaks $[6,7]$, but also unconfined surface flows along straight river segments, bends and confluences [8], as well as impulse waves and tsunamis [4, 9], and water falls [10] among others.

The lattice Boltzmann method (LBM) is an alternative numerical fluid dynamics scheme based on Boltzmann's kinetic equation [11] that represents the flow dynamics at the macroscopic level by incorporating a microscopic kinetic 
approach that also preserves the conservation law $[12,13]$. LBM has been adopted for the characterization of flows in porous media [14] and for multiphase flows [15-17] among others $[18,19]$. The LBM demonstrated to work efficiently for describing the complex physics of non ideal flows and complex geometries [20]. There are also several works investigating the performances of the LBM in simulating shallow free-surface flows $[21,22]$, but with no emphasis on their use for practical engineering applications.

In this work a fully $3 \mathrm{D}$ front-tracking formulation of the LBM, that was originally presented [23] for foam dynamics and that was revisited for free-surface flow applications [2426], is implemented, validated, and compared to the standard NS approach for transient hydraulic flows, including a synthetic case of flow propagation through an urban setting.

The storage effect of urban areas as well as the hydrograph separation effect resulting in different water transmission rates was investigated in several works [27]. Experiments of dam breaks were conducted [28] to investigate the effect of a flood flow propagating to inundate a single building [29]. Nevertheless, the application and potential validation of LBM for urban modeling of flash floods [30] has not been adequately developed yet.

The novel aspect of the proposed work is the investigation for the potential of the LBM to simulate the flooding in highly urbanized areas with specific regard to the detailed characterization of instantaneous discharge variations, like the one caused by dam or dyke breaks or by intense rainfall of flash floods, producing multidimensional street surface flow paths, which is one of the most complex and difficult hazardous phenomena to manage, prevent, and control. The interaction of water flow with anthropogenic features produces complex 3D flow structures that shall be simulated by taking into consideration all the resulting flow physics such as hydraulics jumps, flow constrictions, and back pressure effects. Disregarding those geometric and hydraulic features would result in a misinterpretation of the estimated water levels providing an inaccurate flood hazard and risk characterization.

In this study, given the impossibility of gathering measured data on such phenomena, a comparative analysis of the performance of LBM and NS in simulating flash flood propagation in urban areas is investigated by a comparison with experimental data of dam break hydraulic effects on a synthetic case study represented by an ideal city.

The present document is organized as follows: in the next section, the theoretical and numerical specifications of both LBM and NS are introduced and described. Then, in the case study section, the results of the application of the proposed methods are inserted with specific regard to (1) the test case of the LBM results compared to an analytical solution; (2) the analysis and comparison of LBM and NS codes applied on the simulation of a rapidly varying flow along a simple geometry of a straight river reach; (3) the evaluation and validation of the LBM and NS code application on the flow propagating through a synthetic urban domain by means of comparison with experimental data of a physical model. The last two sections represent, respectively, the discussion and conclusive remarks.

\section{Methods}

2.1. The Navier-Stokes Numerical Scheme. The most general macroscopic model for hydraulic flows may be represented by the incompressible Navier-Stokes equations as follows:

$$
\rho\left(\frac{\partial \mathbf{u}}{\partial t}-\mathbf{F}\right)=-\nabla p+\mu \nabla^{2} \mathbf{u}
$$

where $\rho$ is the fluid density, $\mathbf{F}$ is a specific prescribed body force (i.e., gravity force), and $\mu$ is the fluid dynamic viscosity.

As in hydraulic flows, at least two phases are always present, water and air; the above system of equations must be applied to a multiphase system. The simulation of multiphase flows is a challenging task due to the inherent complexity of the involved phenomena (i.e., moving interfaces with complex topology) and represents one of the leading edges of computational physics.

The following two main approaches are widely used to simulate multiphase flows: the Euler-Lagrange approach and the Euler-Euler approach. The latter approach, usually chosen in hydraulics applications, is based on the assumption that the volume of a phase cannot be occupied by other phases, thus introducing the concept of phase volume fractions as continuous functions of space and time.

For the present work, we employed the so-called volume of fluid (VOF) method, [31, 32] designed for two or more immiscible fluids where only one fluid (i.e., air) is compressible and the position of the interface between the fluids is of interest. The VOF method is a surface-tracking technique applied to a fixed Eulerian mesh, in which a specie transport equation is used to determine the relative volume fraction of the two phases, or phase fraction, in each computational cell. Practically, a single set of Reynolds-averaged Navier-Stokes equations is solved and shared by the fluids, and, for the additional phase, its volume fraction $\gamma$ is tracked throughout the domain.

Therefore, the full set of governing equations for the fluid flow are as follows:

$$
\begin{gathered}
\nabla \cdot \mathbf{u}=0 \\
\frac{\partial \rho \mathbf{u}}{\partial t}+\nabla \cdot(\rho \mathbf{u u})-\nabla \cdot\left(\left(\mu+\mu_{t}\right) \mathbf{S}\right)=-\nabla p+\rho \mathbf{g}+\sigma K \frac{\nabla \gamma}{|\nabla \gamma|} \\
\frac{\partial \gamma}{\partial t}+\nabla \cdot(\mathbf{u} \gamma)=0
\end{gathered}
$$

where $\mathbf{u}$ is the velocity vector field, $p$ is the pressure field, $\mu_{t}$ is the turbulent eddy viscosity, $\mathbf{S}$ is the strain rate tensor defined by $\mathbf{S}=\left(\nabla \mathbf{u}+\nabla \mathbf{u}^{T}\right) / 2, \sigma$ is the surface tension, and $K$ is the surface curvature.

The nature of the VOF method means that an interface between the species is not explicitly computed, but rather emerges as a property of the phase fraction field. Since the phase fraction can have any value between 0 and 1 , the interface is never sharply defined, but occupies a volume around the region where a sharp interface should exist. 
TABLE 1: Velocity vectors for the D3Q19 models.

\begin{tabular}{lccc}
\hline $\mathbf{c}_{\mathbf{i}}$ & Direction & Number of cells & $f_{i}^{\mathrm{eq} 0}$ \\
\hline 0 & 0 & 1 & $f_{1}^{\mathrm{eq} 0}$ \\
$\mathrm{c}$ & $1, \ldots, 6$ & 6 & $f_{2}^{\mathrm{eq} 0}$ \\
\hline$c \cdot \sqrt{2}$ & $7, \ldots, 18$ & 12 & $f_{3}^{\mathrm{eq} 0}$ \\
\hline
\end{tabular}

The model used in this work is based on an open source computational fluid dynamics (CFD) platform named OpenFOAM [33], freely available on the Internet. OpenFOAM, primarily designed for problems in continuum mechanics, uses the tensorial approach and object-oriented techniques. It provides a fundamental platform to write $\mathrm{C}++$ new solvers for different problems as long as the problem can be written in tensorial partial differential equation form.

2.2. The LBM Numerical Scheme. This section introduces the theoretical background of the proposed LBM with specific regard to the following:

(i) the single-phase LBM and the boundary conditions,

(ii) the front tracking concept,

that are, respectively, inserted in the two following sections.

2.2.1. Single-Phase Lattice Boltzmann Method and Boundary Conditions. Artificial and natural fluvial hydrodynamics may be simulated at the macroscopic scale using the LBM that is structured as a mesoscopic (i.e., scaled larger than micro and smaller than macro) method. LBM treats the water mass by analysing its density probability function, $f_{i}\left(\mathbf{x}, \mathbf{c}_{\mathbf{i}}, t\right)$, where $\mathbf{x}$ is the particle spatial location within the lattice domain, $t$ is the time variable, and $c_{i}$ is the kinematic component. The dynamic of the particle distribution function is described in (3) that represents a discrete lattice Boltzmann equation, representing the evolution in time of the fluid flow by providing a numerical solution of the modification of the density probability function:

$$
\begin{aligned}
f_{i}(\mathbf{x} & \left.+\mathbf{c}_{\mathbf{i}} \Delta t, t+\Delta t\right)-f_{i}(\mathbf{x}, t) \\
& +\Delta t \cdot \mathbf{F}_{\mathbf{i}}=C_{i}(\mathbf{x}, t), \quad i=1, \ldots, b,
\end{aligned}
$$

where the left and right terms represent, respectively, the molecular free-streaming and the particle collisions. $\mathbf{F}_{\mathbf{i}}$ is an additional term accounting for internal (e.g., collision) and external forcing processes (e.g., electric and magnetic fields or gravity).

For water-driven processes, it is reasonable to assume a discrete set of directions along which particles may route and, as a consequence, $\boldsymbol{c}_{\mathbf{i}}$ is restricted to a maximum of 7 to 9 for 2D or 19 directions for 3D flows. This 19-direction discretized cubic lattice model implemented here (namely, D3Q19) is characterized by the set of predefined velocity vectors schematically represented in Figure 1 and reported in Table 1.

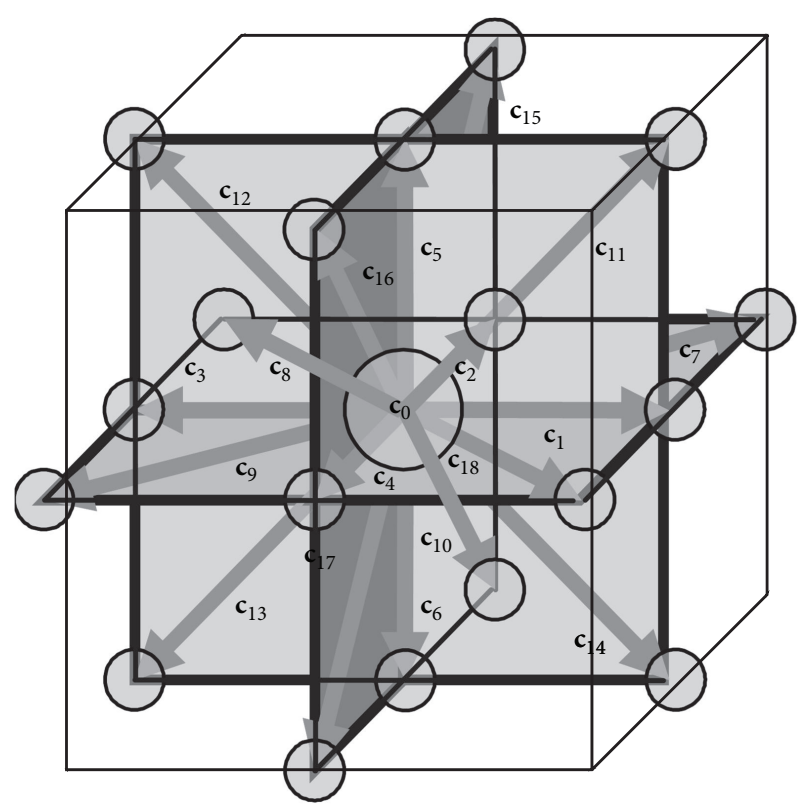

FIGURE 1: D3Q19 velocity model.

The collision component is integrated by implementing the Bhatnagar-Gross-Krook Model (BGK) [34]

$$
C_{i}(\mathbf{x}, t)=-\frac{\Delta t}{\tau}\left[f_{i}(\mathbf{x}, t)-f_{i}^{\mathrm{eq}}(\mathbf{x}, t)\right]
$$

that characterizes the density modifications caused by particle collisions as related to dynamic modifications from the local equilibrium $f_{i}^{\text {eq }}[35,36]$.

If the relative Knudsen and Mach numbers are small enough, this formulation is able to represent the dynamics of a fluid with pressure $P=c_{s}^{2} \rho$ and a kinematic viscosity $v=c_{s}^{2}(\tau-\Delta t / 2)$, where $c_{s}$ is the lattice sound velocity [12].

The proposed numerical approach is implemented on a structured Cartesian lattice (i.e., mesh) where the particles may only reside on the mesh nodes.

In the proposed model boundary nodes are solved using the bounce-back scheme meaning that particles colliding with a stationary wall simply reverse their momentum [12, 37]. In particular, the half-way bounce-back scheme is here applied by posing the reflecting wall at a medium distance between boundary nodes and the physical walls [38]. As a result, the proposed model can simulate both solid features (e.g., walls, levees, etc.) of known geometry or open boundaries of predefined velocity, discharge, or pressure function.

The turbulence flow field is implemented in the proposed LBM model by employing a Large Eddy Simulation (LES) approach [39] that characterizes the flow field simulating large-scale structures (resolved grid scales), which are assumed to be mostly predominating in defining the transport of mass and momentum, and not directly simulating the small-scale local effects. The definition of the boundary between small and large processes is based on the Smagorinsky model [40]. 
2.2.2. Front-Tracking Model. In the Front-Tracking (FT) formulation proposed here [23], the interface is treated as a zero thickness, mathematical surface, across which the density field jumps from the light to the dense phase and vice versa and is tracked throughout the computational domain through an additional scalar variable (i.e., liquid mass fraction). A single set of LB equations is solved for both the heavy fluids with respect to the light fluids (i.e., gas) in agreement with the front-tracking methodology that do not aim at providing any description of the physics of the phase-transition between the two phases. The main difference, which also represents the main advantage of the LBM front-tracking approach, with respect to the traditional continuum methods, is that the single-species transport equation, used in NavierStokes/VOF model to determine the relative volume fraction of the two phases in each computational cell, is not needed, since the free-surface tracking is automatically performed by advancing the fluid elements. In practical terms, only the liquid phase flow is numerically solved, while the gas characterization is neglected, and an additional variable $\varepsilon$ (i.e., the liquid volume fraction) is structured to track the free surface throughout the computational domain. This variable is numerically solved using the following equation:

$$
\varepsilon(\mathbf{x}, t)=\frac{m(\mathbf{x}, t)}{\rho(\mathbf{x}, t)}: \begin{cases}\varepsilon(\mathbf{x})=0, & \forall \mathbf{x} \in G, \\ \varepsilon(\mathbf{x})=1, & \forall \mathbf{x} \in L, \\ 0<\varepsilon(\mathbf{x})<1, & \forall \mathbf{x} \in I,\end{cases}
$$

in which $G, L$, and $I$ represent respectively empty (gas), liquid, and interface cells.

The computation scheme is integrated numerically in three steps as follows: (1) interface positioning and flow mass motion update defined by analysing cell fluxes; (2) definition of the boundary conditions at the interface to separate the gas and liquid state; (3) update of cell type. As a result, interface cells defined by the $\mathbf{x}$ position and time $t$ are defined as follows:

$$
\Delta m_{i}= \begin{cases}0, & \forall \mathbf{x} \in G, \\ f_{i}^{\mathrm{pc}}\left(\mathbf{x}+\mathbf{c}_{\mathbf{i}} \Delta t, t\right)-f_{-i}^{\mathrm{pc}}(\mathbf{x}, t), & \forall \mathbf{x} \in L, \\ \left(\frac{\varepsilon(\mathbf{x}, t)+\varepsilon\left(\mathbf{x}+\mathbf{c}_{\mathbf{i}} \Delta t, t\right)}{2}\right) & \\ \quad \times\left[f_{i}^{\mathrm{pc}}\left(\mathbf{x}+\mathbf{c}_{\mathbf{i}} \Delta t, t\right)-f_{-i}^{\mathrm{pc}}(\mathbf{x}, t)\right], & \forall \mathbf{x} \in I,\end{cases}
$$

in which pc characterizes the postcollision state and the sign is related to the mass direction where $-i$ has opposite direction with respect to $i$. Mass dynamics in space and time are defined as

$$
m(\mathbf{x}, t+\Delta t)=m(\mathbf{x}, t)+\sum_{i=1}^{18} \Delta m_{i}(\mathbf{x}, t) .
$$

Combining (6) and (7), the continuity principium is in respect with the condition that direct modification of state, from liquid to gas or vice versa, is not permitted outside the interface. In fact, liquid and gas cells are only allowed to transform into interface cells, whereas interface cells can be

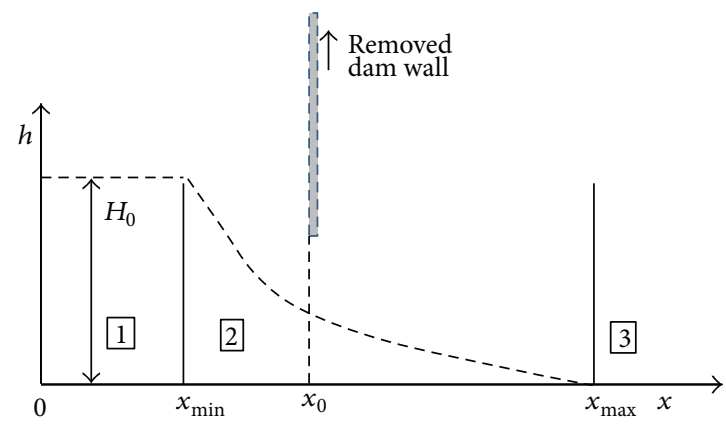

FIGURE 2: Schematic representation of the water surface profile for a $20 \mathrm{~m}$ long straight channel dam break.

transformed into both gas and liquid cells. In this way, mass and density are completely decoupled and, as a results, mass evolution does not affect the particle distribution functions.

\section{Numerical Results for Three Case Studies}

The proposed LBM and NS are applied for the following three test cases:

(a) the free-surface flow routed along a straight channel, with LBM modeling results that are validated by comparison with two different types of analytical solutions;

(b) the free-surface flow routed along a straight channel deriving from the partial asymmetrical collapse of a vertical dam embankment with results that are compared in terms of hydraulics and computational efficiency;

(c) the simulation of a severe transient flow through a synthetic urban district.

The three tests are hereafter presented in detail.

3.1. Test (a): Fast Flow Propagating along a Straight Channel. The routing of a fast moving flow resulting from a dam break, propagated through a $20 \mathrm{~m}$ long straight channel (see Figure 2), is simulated, and the numerical results are compared to the analytical solution provided by Ritter [41].

The channel bed is horizontal. Bottom and wall friction is set to null. The numerical mesh is made up of 2,000 cubic elements with a grid-spacing of $0.01 \mathrm{~m}$. At time zero, a $0.5 \mathrm{~m}$ by $7 \mathrm{~m}$ volume of water ideally positioned at the most, upstream section of the channel is released instantaneously and two resulting waves propagate, respectively, downstream and upstream back to the reservoir. At a given time $t$ from the start of the simulation (i.e., dam break/breach or ideal vertical wall removal), three different regions are defined (Figure 2) as follows:

(1) for $x<x_{\min }$, we have $h=H_{0}=0.5 \mathrm{~m}$, 


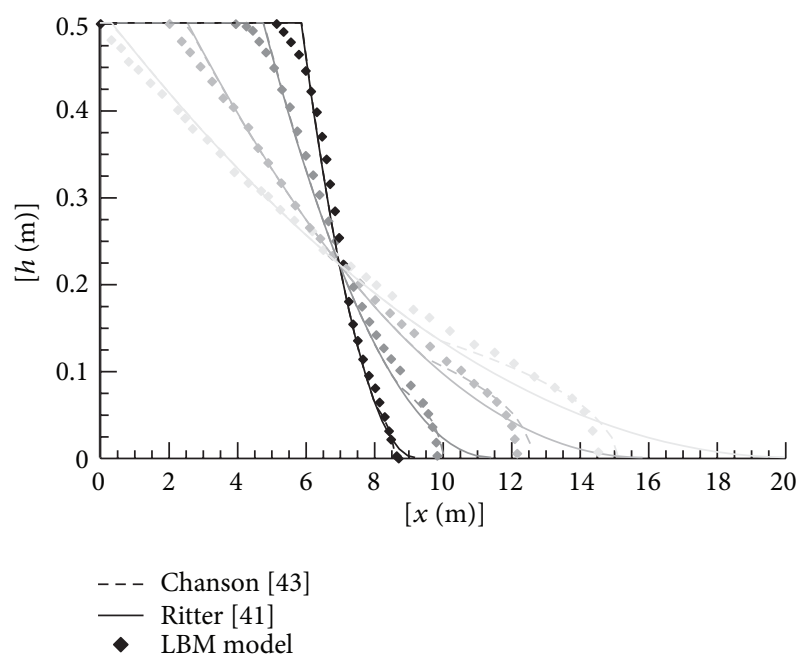

FIGURE 3: LBM simulation results, analytical solution, and experimental data for the $20 \mathrm{~m}$ long straight channel dam break at times $0.5 \mathrm{~s}, 1 \mathrm{~s}, 2 \mathrm{~s}$, and $3 \mathrm{~s}$.

(2) for $x_{\min }<x<x_{\max }$, the water surface profile is parabolic

$$
\begin{gathered}
h=\frac{H_{0}}{\left(x_{\min }-x_{\max }\right)^{2}}\left(x^{2}-2 x_{\max } x+x_{\max }^{2}\right), \\
x_{\min }=-t \sqrt{g H_{0}}+x_{0}, \\
x_{\max }=2 t \sqrt{g H_{0}}+x_{0},
\end{gathered}
$$

(3) for $x>x_{\max }$, we have $h=0$,

where $h$ is the water elevation. Figure 3 shows the agreement between numerical and analytical data apart some minor expected differences at the boundaries [42]. In fact, where Ritter's analytical scheme is not suited for this comparison, other methods were selected like the Chanson's solution [43]:

$$
\begin{gathered}
\frac{8}{3} \frac{1}{f} \frac{(1-U / 2)^{3}}{U^{2}}=\frac{t}{\sqrt{g / H_{0}}}, \\
\frac{x_{s}}{H_{0}}=\frac{x_{0}}{H_{0}}+\left(\frac{3}{2} U-1\right) \frac{t}{\sqrt{g / H_{0}}}+\frac{4}{f U^{2}}\left(1-\frac{U}{2}\right)^{4}, \\
\frac{h}{H_{0}}=\frac{1}{9}\left(2-\frac{\left(x-x_{0}\right)}{H_{0}\left(t / \sqrt{g / H_{0}}\right)}\right)^{2} \\
\text { if }-\frac{t}{\sqrt{g / H_{0}}} \leq \frac{\left(x-x_{0}\right)}{H_{0}} \leq\left(\frac{3}{2} U-1\right) \frac{t}{\sqrt{g / H_{0}}} \\
\quad \text { if }\left(\frac{\left.\frac{3}{2} U-1\right) \frac{t}{\sqrt{H_{0}}}=}{U^{2} \frac{\left(x_{s}-x\right)}{H_{0}}} \leq \frac{\left(x-x_{0}\right)}{H_{0}} \leq \frac{\left(x_{s}-x_{0}\right)}{H_{0}},\right.
\end{gathered}
$$

that is also plotted in Figure 3.

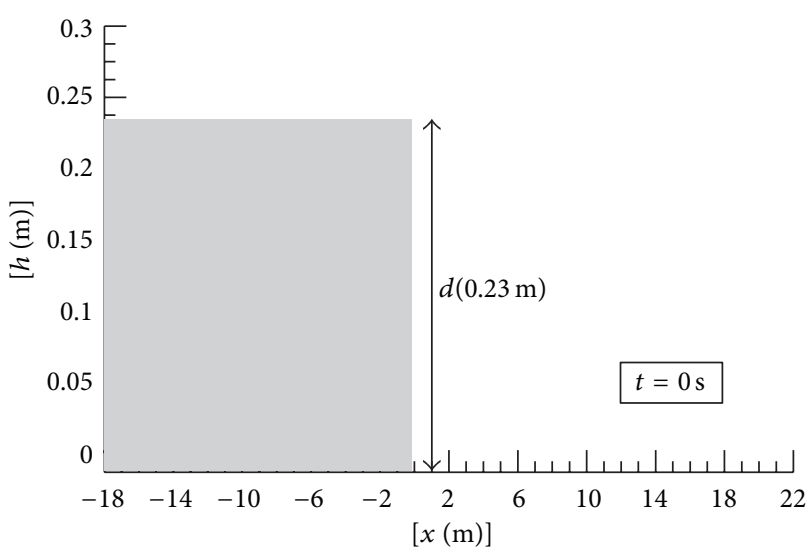

(a)

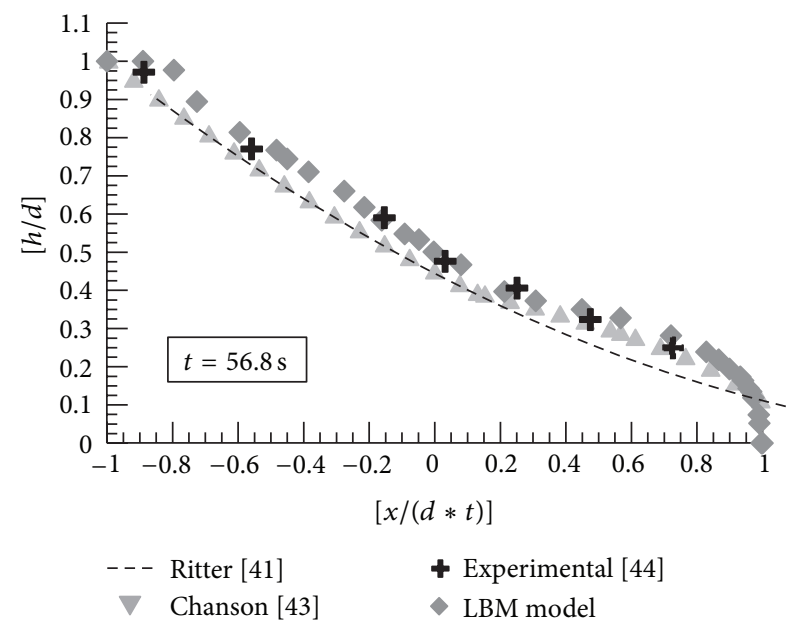

(b)

FIgURE 4: Comparison between numerical LBM and analytical and experimental results at time $56.8 \mathrm{~s}$ for the $40 \mathrm{~m}$ long straight channel dam break.

To further prove the validity of the code, a dam break flow propagating along a $40 \mathrm{~m}$ long straight channel is simulated. At time zero, a $0.23 \mathrm{~m}$ by $18 \mathrm{~m}$ water volume is released instantaneously. In Figure 4, LBM numerical results are compared to the Ritter [41] and Chanson [43] as well as to the experimental measurements provided by [44]. The comparison is performed in terms of dimensionless instantaneous free-surface profiles $(h / d)$ between numerical and analytical data (assuming $f=0.02$ ).

3.2. Test (b): Asymmetrical Dam Break-LBM and NS Numerical Models. The second test is characterized by the simulation of a submersion wave caused by the partial collapse of a nonsymmetrical sluice gate (or partial asymmetric dam breach). The spatial 3D computational domain is $200 \mathrm{~m} \times$ $200 \mathrm{~m} \times 20 \mathrm{~m}$, where the boundaries of the domain are impermeable walls. The nonuniform initial condition for the liquid phase fraction is specified: the water surface level is initially $10 \mathrm{~m}$ for the upstream section and $5 \mathrm{~m}$ for the downstream section. An unsteady flow is generated by 

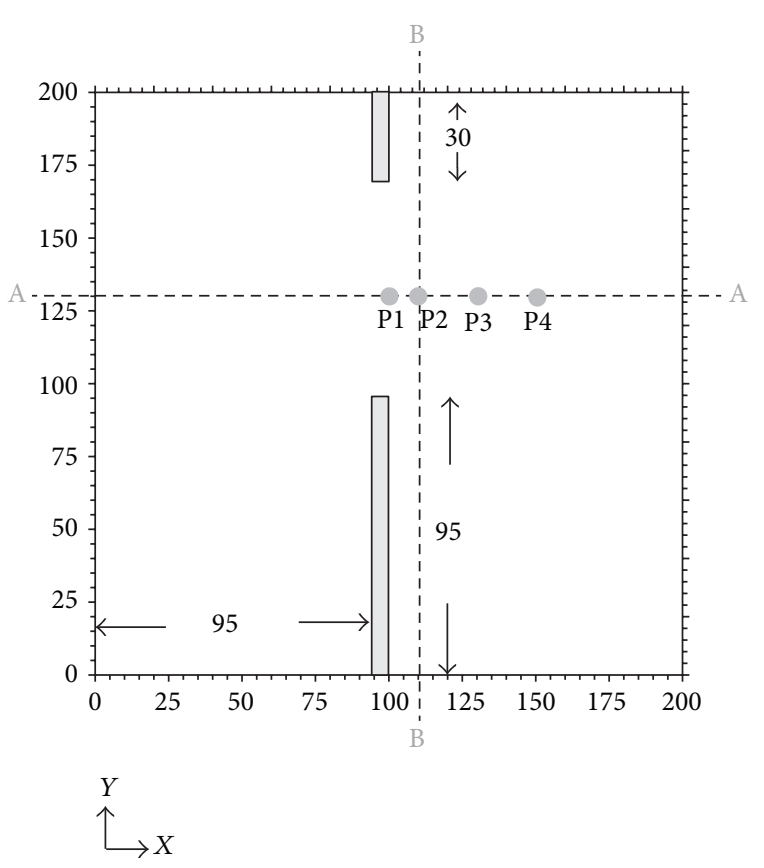

FIGURE 5: Geometric schematization in plan view for test (b). Sections A-A and B-B and Points P1 $(100,130)$, P2 $(110,130)$, P3 (130, $130)$, and $\mathrm{P} 4(150,130)$ are shown. All dimensions are in $\mathrm{m}$.

the instantaneous collapse of an asymmetrical $75 \mathrm{~m}$ long portion of the dam (see Figure 5). The bottom is flat, and ground resistance to the motion is neglected.

Numerical results of several authors are available in the literature for this case $[6,45,46]$, that is, a standard benchmark for hydraulic models in dam break applications. We chose to compare the LBM and NS, described in the previous section, in terms of computational efficiency. Calculations have been performed with a time step of $0.01 \mathrm{~s}$ on a computational mesh of 800,000 nodes (i.e., lattice sites for the LBM). Figure 6 shows the comparison of the computed water level 7.2 seconds after the breach, when the flow reaches the left side of the tank, while Figure 7 shows the simulated water surface levels along sections A-A (longitudinal $Y=130 \mathrm{~m}$ ) and $\mathrm{B}-\mathrm{B}$ (transverse $X=110 \mathrm{~m}$ ). Water level hydrographs at selected points of the domain have been also evaluated, and Figure 8 shows the results obtained for points $\mathrm{P} 1$ and $\mathrm{P} 3$ with both LBM and NS approaches.

Results show that NS and LBM, using the same grid resolution, are in good agreement in terms of water heights and hydrograph, along the section AA, while the results for the section BB (Figure 7(b)) are remarkably different especially in terms of water depths. This is expected with specific regard to the central part of the domain where the dam break originates and the flow is significantly $3 \mathrm{D}$, while in the other portions of the domain, through which the emptying of the system applies (from the noncollapsing area towards where the breach is) in the direction of the flow that is parallel to the gate, the two models give different results with greater water depths evaluated by NS.

From the computational point of view, using the same hardware, the LBM model has completed the tests in one-fifth
TABLE 2: Computational efficiency of LBM and NS.

\begin{tabular}{lccc}
\hline $\begin{array}{l}\text { Mathematical } \\
\text { Model }\end{array}$ & $\Delta x$ & $\begin{array}{c}\text { Computational } \\
\text { time }(\mathrm{s})\end{array}$ & $\begin{array}{c}\text { Simulation } \\
\text { time }(\mathrm{s})\end{array}$ \\
\hline NS & 1.0 & 6941 & 7.2 \\
LBM & 1.0 & 1512 & 7.2 \\
LBM & 0.8 & 3011.04 & 7.2 \\
LBM & 0.6 & 4676.4 & 7.2 \\
LBM & 0.4 & 6350 & 7.2 \\
LBM & 0.3 & 7300.08 & 7.2 \\
\hline
\end{tabular}

of the time needed by the NS. Results are summarized in Table 2 that also shows that the LBM code is able to provide a higher level of details of the flow pattern using the same computational time or to provide the same accuracy and details in less time. Figure 9, synthesizing the LBM versus NS computational efficiency comparison test, shows that the LBM model can use a mesh $65 \%$ denser than NS one $(\Delta X(\mathrm{LBM})=0.35 \mathrm{~cm}, \Delta X(\mathrm{NS})=1 \mathrm{~m})$ for the same computational time.

\subsection{Test (c): Severe Transient Flow through a Synthetic Urban}

District. This test presents the simulation of the effects of a severe transient flow on a synthetic urban area. This case study replicates the experiment developed at the Hydraulic Laboratory of the Civil and Environmental Engineering Department of the Université Catholique de Louvain in Belgium [47]. The geometric configuration is characterized by a regular distribution of rectangular obstacles (buildings) intercepting the flow in geometric domain. The realistic average ratio between building and street widths of 1 to 3 was selected by interpreting a real urban configuration (the aerial view of Brussels in Belgium). Buildings' heights are simulated as high enough in order not to be overtopped by the flow. The experimental physical setup consists of a $36 \mathrm{~m}$ long by $3.6 \mathrm{~m}$ wide channel, with a flat profile (Figure 10). Buildings, wooden parallelepipeds of $30 \mathrm{~cm}$ by $30 \mathrm{~cm}$, and streets that are $10 \mathrm{~cm}$ wide are positioned along the flow direction. The initial reservoir water level is $0.40 \mathrm{~m}$ upstream. Water surface measurements were performed [47] by means of several resistive level gauges for estimating the water surface geometric and dynamic properties (depths and surface velocity fields).

The aim of this test is to investigate the potential of LBM and NS to correctly represent the complex dynamics of transient flows. In particular, the physics of interest is related to those flood phenomena propagated through urban setting for which an initial severe transient phase is often followed by a slow transient that could approximate under certain steady state conditions. Nevertheless, since the first instants correspond to a severe transient flow involving specific flow features characterized by high velocities and transcritical flows, wave impacts on structures, or higher water rise following wave reflection against a structure, the LBM and NS are challenged to provide such unsteady irregular behavior followed by the regular steady conditions of the following sequences. 


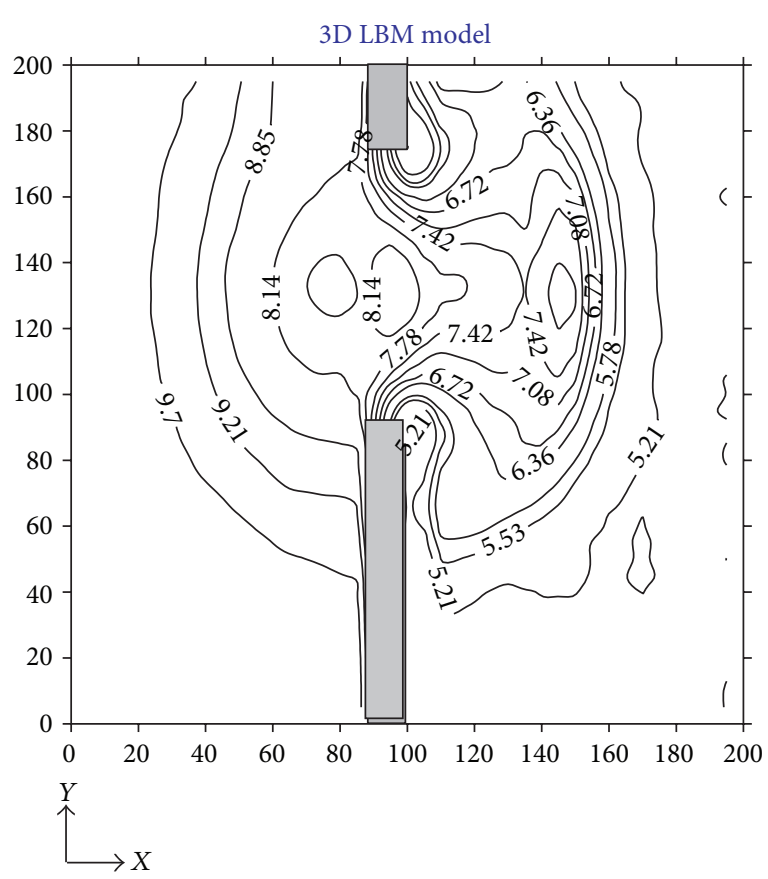

(a)

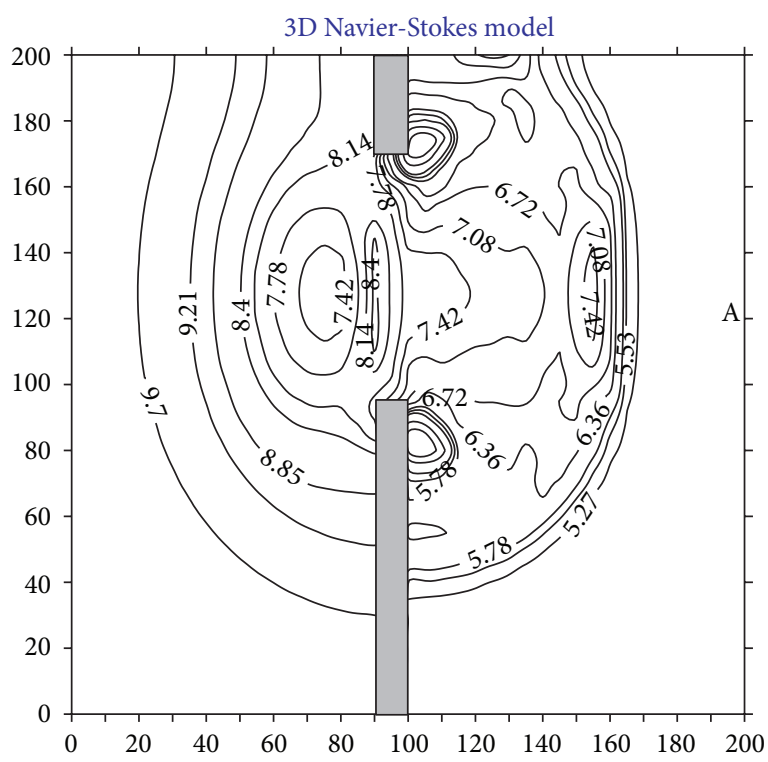

(b)

FIGURE 6: Comparison of the computed water level 7.2 seconds after the breach-LBM versus and NS are, respectively, plotted in the left and right.

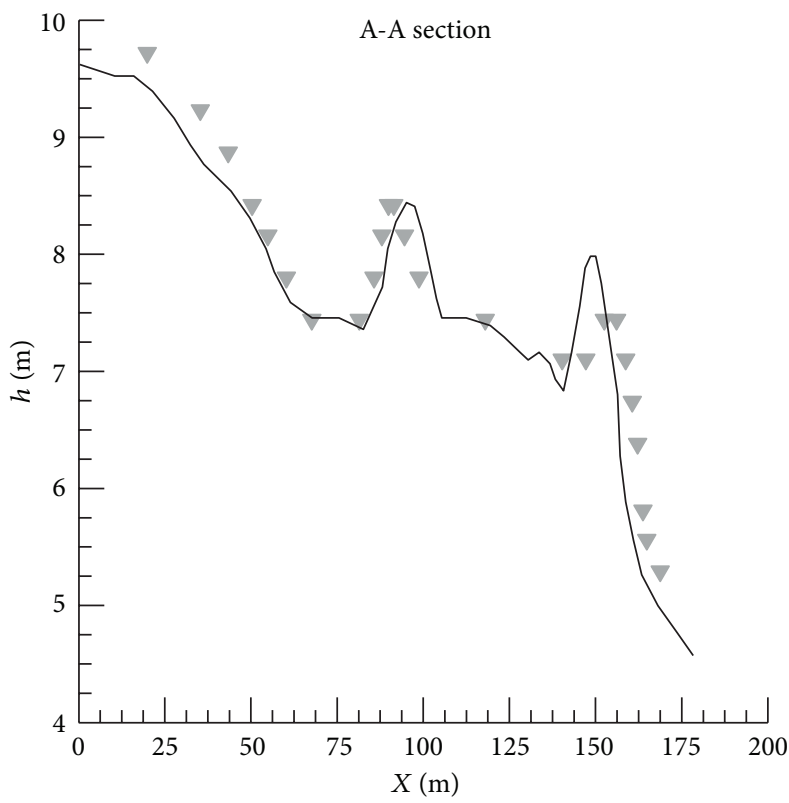

$\checkmark$ NS model

- LBM model

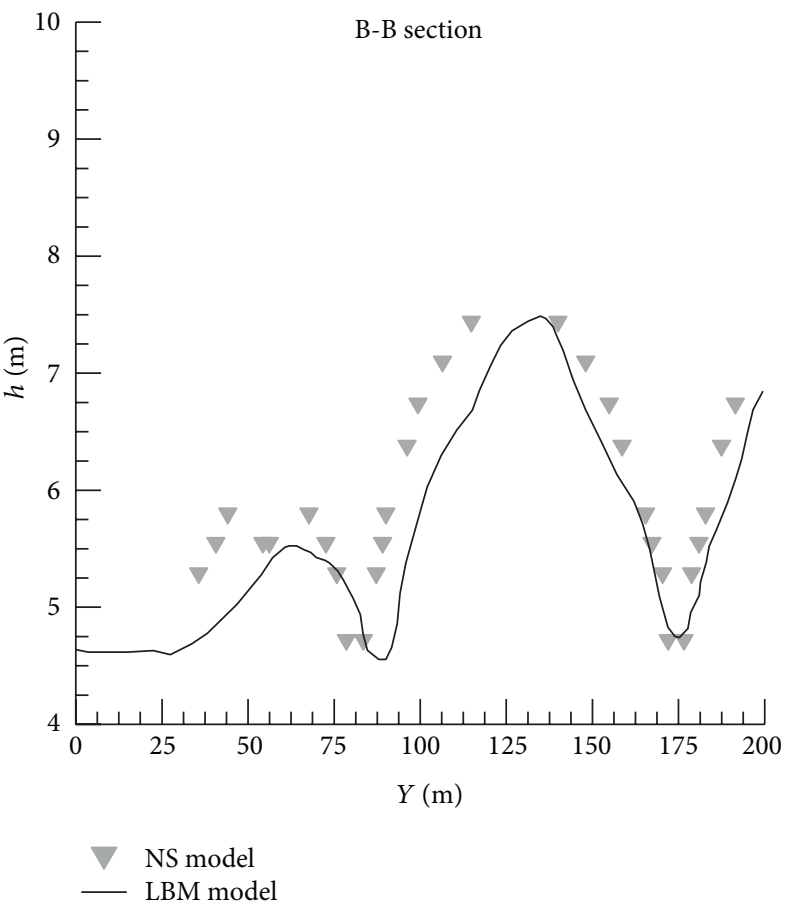

(b)

Figure 7: Comparison between water surface elevations obtained with LBM and NS in the A-A and B-B sections at 7.2 seconds after the failure. 


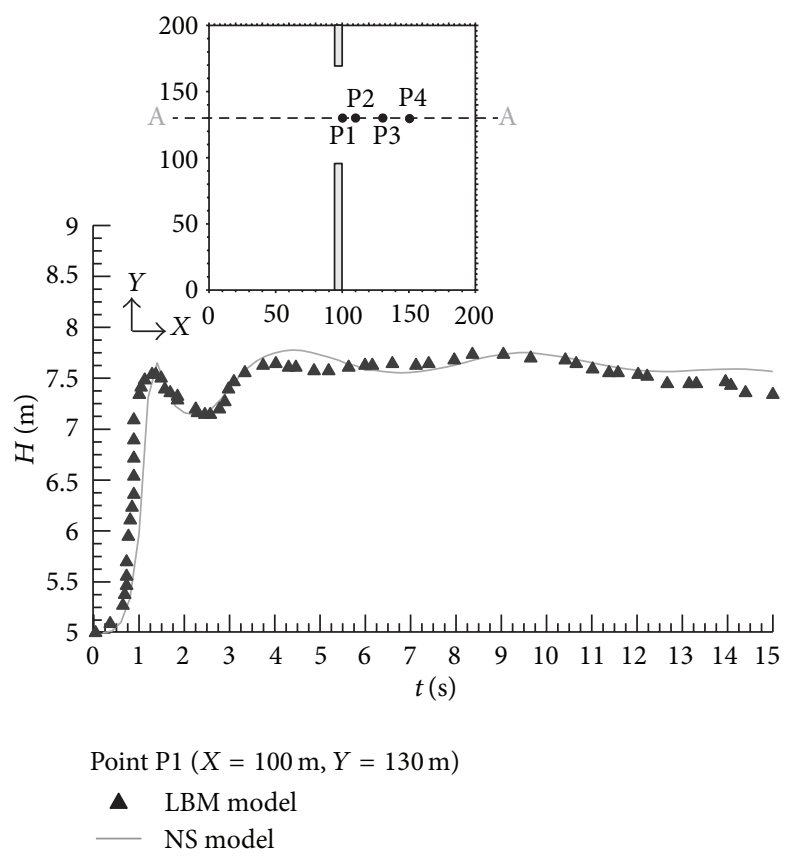

(a)

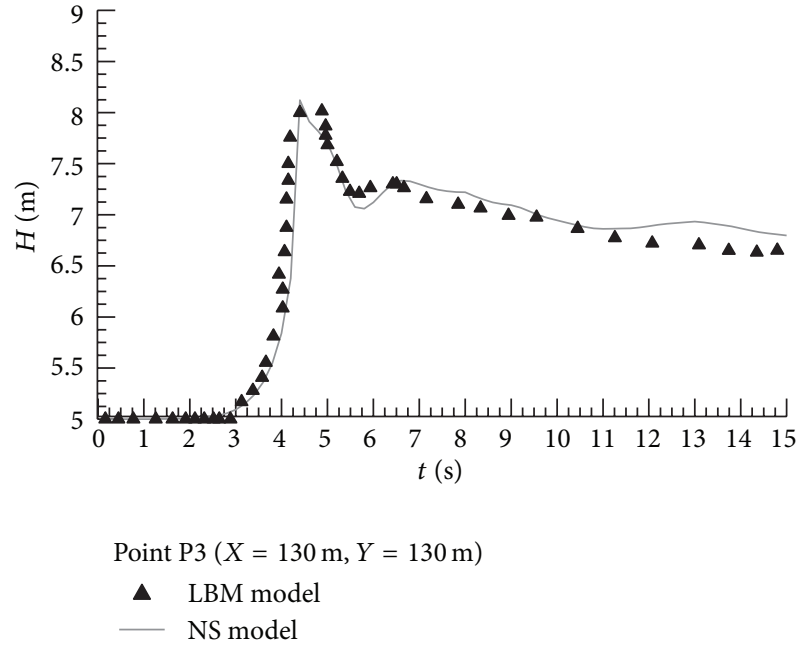

(b)

FIgURE 8: Water level hydrographs at points P1 $(100,130)$ and P3 $(130,130)$ for test $(b)$. Continuous line LBM, scatter points for NS.

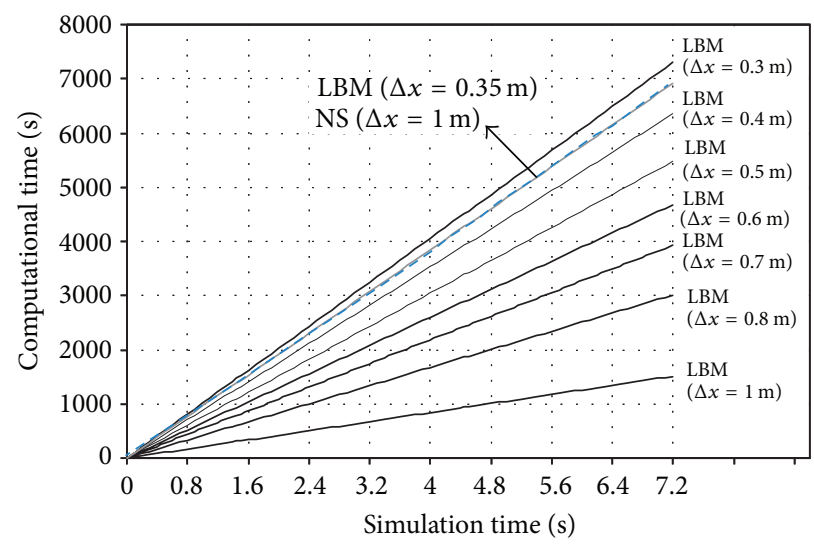

FIGURE 9: Computational comparison test for and LBM models for test (b).

3.3.1. Simulation Setup-Mesoscopic Approach Using LBM. Due to the large-scale of the domain in horizontal XY plane, the test case has been replicated using a computational mesh of cubes of $0.025 \mathrm{~m}$ resolution producing a total number of $4,976,640$ nodes. The downstream region has been represented assigning a liquid volume fraction of 0.5 to the first vertical row of nodes. Considering the dimension of the domain, the mesh is quite rough and only few computational nodes describe the streets that are only $0.1 \mathrm{~m}$ wide.

3.3.2. Simulation Setup-Continuous Approach Using NS. For all simulations, the computational domain is the same with the following dimensions: $36 \mathrm{~m}$ long, $3.6 \mathrm{~m}$ wide, and $1 \mathrm{~m}$ high. Five scenarios have been developed for the NS simulation, varying the resolution of the mesh in the three directions. The computational mesh for each scenario derives from the intersection and refinement of the mesh composed of hexahedra (configuration A, see Table 3) with the 3D surface of the synthetic urban setting (Figure 11). In configuration A a grid variation along the vertical with a ratio of 1 to 10 is used. The result of such a refinement process is represented by configuration B (see Table 3). For the five scenarios, M1 to M5, the diverse grid resolutions lead to discrepancies between the numerical results and physical measurements both inside and outside the city. The comparison between experimental data and numerical results provided by the LBM and the NS, scenarios M1, M2, M5, for the section $y=2 \mathrm{~m}$ at $t=5,6$ and $10 \mathrm{~s}$ after the break are presented in Figure 12.

The free-surface profile includes a hydraulic jump at $5 \mathrm{~s}$ after the break. In the graphs for $t=5 \mathrm{~s}$ and $t=6 \mathrm{~s}$, when the flow depth in the city is still low, the flow reflection against the buildings is clearly visible for both LBM and NS simulations, 
TABLE 3: Geometric characteristics of computational meshes adopted for scenarios M1, M2, M3, M4, and M5.

\begin{tabular}{|c|c|c|c|c|c|}
\hline & M1 & M2 & M3 & M4 & M5 \\
\hline Number of TOT cells & $3.24 E+04$ & $2.59 E+05$ & $5.18 E+05$ & $1.04 E+06$ & $2.07 E+06$ \\
\hline Min volume cell $\left(\mathrm{m}^{3}\right)$ & $9.79 E-04$ & $1.25 E-04$ & $6.33 E-05$ & $3.16 E-05$ & $1.58 E-05$ \\
\hline Max volume cell $\left(\mathrm{m}^{3}\right)$ & $9.79 E-03$ & $1.25 E-03$ & $6.33 E-04$ & $3.16 E-04$ & $1.58 E-04$ \\
\hline \multirow{2}{*}{ Number of points } & $3.78 E+04$ & $2.80 E+05$ & $5.48 E+05$ & $1.08 E+06$ & $2.16 E+06$ \\
\hline & $(180 \times 18 \times 10)$ & $(360 \times 36 \times 20)$ & $(360 \times 36 \times 40)$ & $(360 \times 72 \times 40)$ & $(720 \times 72 \times 40)$ \\
\hline Number of faces & $1.02 E+05$ & $7.98 E+05$ & $1.58 E+06$ & $3.15 E+06$ & $6.30 E+06$ \\
\hline Number of TOT cells & $6.16 E+04$ & $4.01 E+05$ & $7.96 E+05$ & $1.12 E+06$ & $2.02 E+06$ \\
\hline Min volume cell & $6.10 E-06$ & $1.50 E-06$ & $7.70 E-07$ & $3.10 E-06$ & $1.25 E-05$ \\
\hline Max volume cell & $9.79 E-03$ & $1.25 E-03$ & $6.33 E-04$ & $3.16 E-04$ & $1.58 E-04$ \\
\hline Number of points & $8.35 E+04$ & $4.84 E+05$ & $9.37 E+05$ & $1.21 E+06$ & $2.12 E+06$ \\
\hline Number of faces & $2.07 E+05$ & $1.29 E+06$ & $2.53 E+06$ & $3.44 E+06$ & $6.17 E+06$ \\
\hline
\end{tabular}

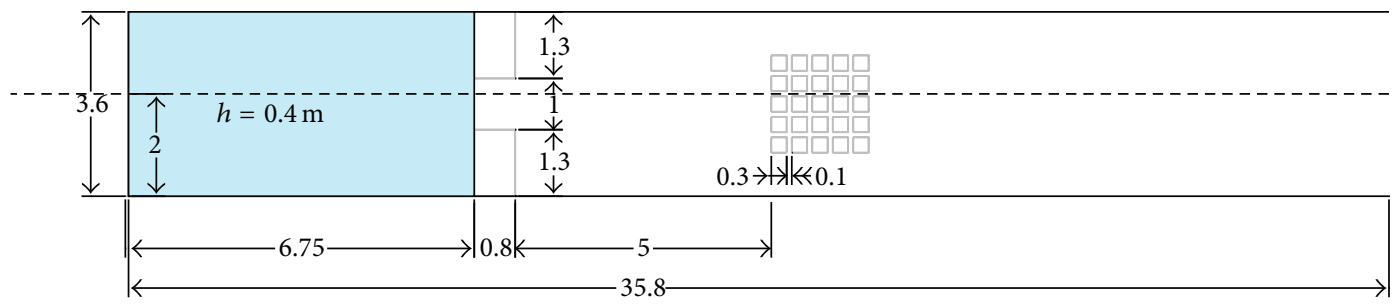

FIGURE 10: Schematic representation of experimental and computational domain.

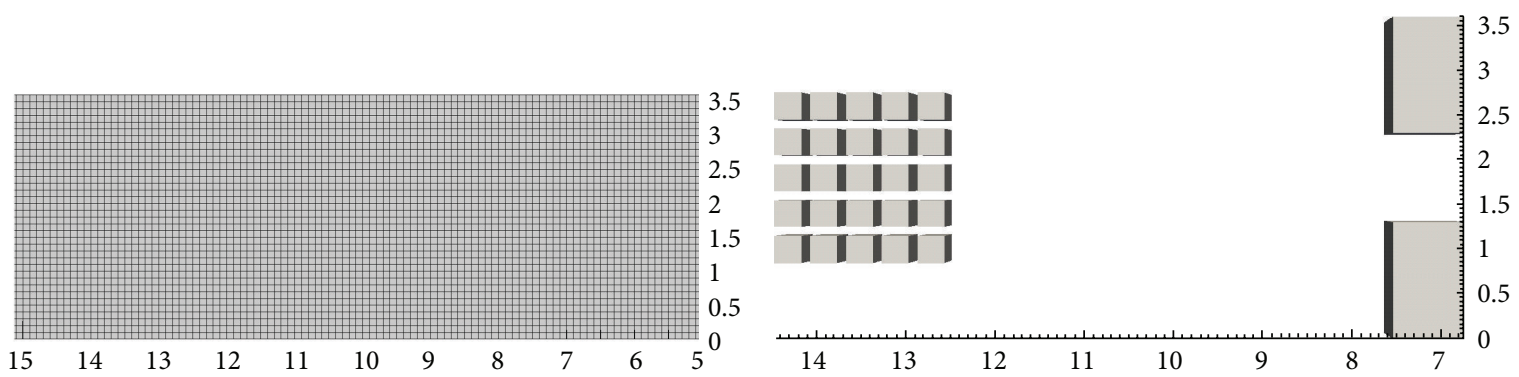

(a)

(b)

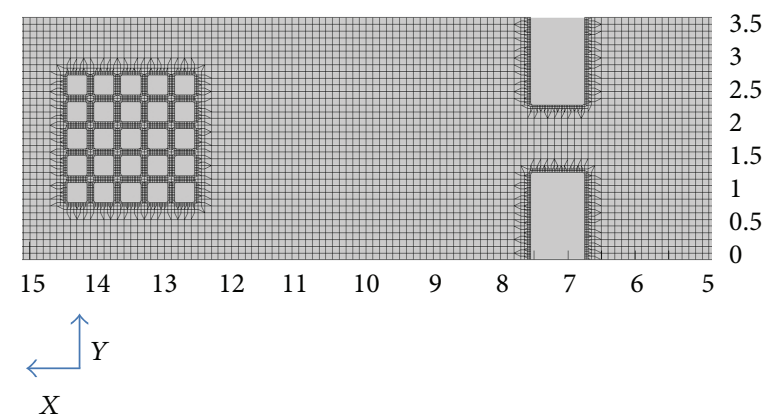

(c)

FIGURE 11: Computational mesh definition procedure. Example of intersection between 3D obstacle surface and original mesh. 


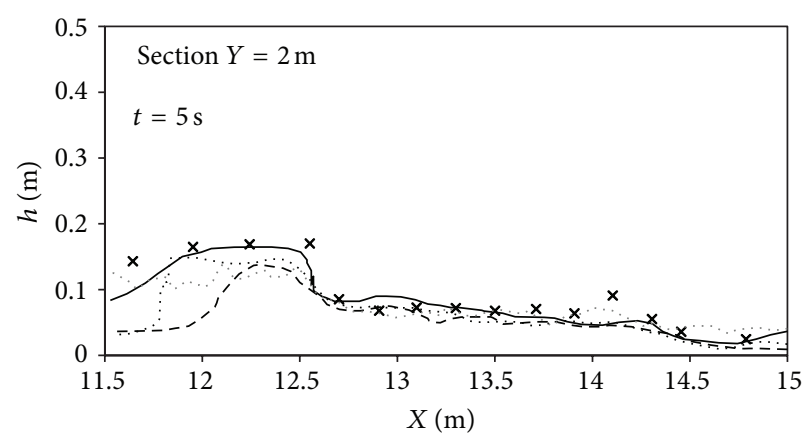

(a)

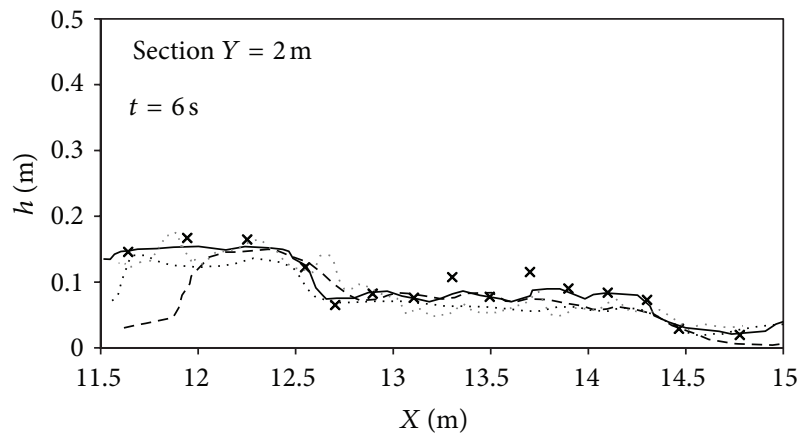

(b)

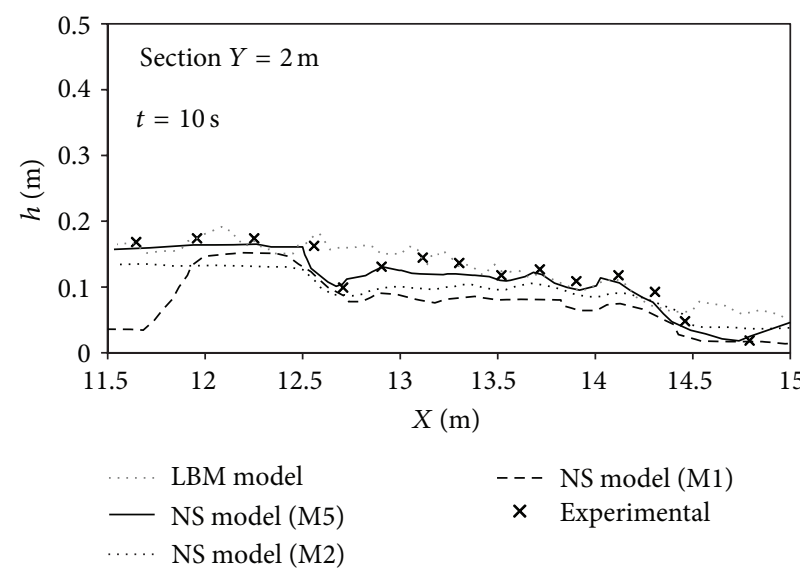

(c)

FIGURE 12: Comparison between experimental and simulated free-surface value for the section $Y=2 \mathrm{~m}$ for scenarios M1, M5 of continuous approach, and LBM for $t=5,6$, and $10 \mathrm{~s}$ after the break.

and the LBM model correctly predicts the free surface at low $x$ values as the NS model with the finest mesh. At $t=10 \mathrm{~s}$, the upstream hydraulic jump is still present, but the water level has significantly increased in the streets. The flow in the streets evolves from supercritical Fr $>1$ (with a control section near the street entrance) to subcritical (with a control section at the street exit).

The overall agreement between the numerical and experimental results is promising for the use of the mesoscopic numerical model for replicating the complex physics of rapidly varying flows in such a dense urban area, even if, due to the mesh resolution, results of lattice-based simulations are less accurate than the NS because of the mesh refinement (especially for M5). Analyzing the effect of mesh refinement, the obstacle mesh refinement of M3-M5 is able to better reproduce the unsteady hydraulic features and flow processes like hydraulics jumps, while the coarser meshes of M1-M2 are characterized by some errors where the flow behavior is irregular.

For LBM at the time steps $t=5$ and $6 \mathrm{~s}$, significant differences appear between the two numerical models. For example, the depression and the hydraulic jump at the entrance of the urban district $(x=5.20 \mathrm{~m})$ is absent in the LBM coarse mesh result. One limitation of the used LBM code is that the computational mesh is not refined locally for example in a single area of interest. This disadvantage with respect to the used NS model clearly indicates the utility of employing mesh refinement techniques, as those developed for standard LBM [48].

Figure 13 shows the results at $t=4$ seconds for 5 different meshes, while in Figure 14 snapshots of free-surface evolution at different time steps $(0,2,4,6,8$, and $10 \mathrm{~s})$ are inserted. Figure 15 reports the value of velocity magnitude for the same time steps.

Observations indicated that the flow rises at the city front before entering the streets, after the wave impact, a phenomenon that is similar to the impact against a single obstacle rather than a series of obstacles. A hydraulic jump is represented at the impact section (Figure 14), with the water level that is locally higher with respect to the same case without buildings, while a wake zone is developed immediately downstream of the city.

\section{Conclusions}

In this study an experimental fully 3D flow modeling framework is implemented to numerically simulate the unsteady irregular flows originating from dam breaks with specific regard to the complex interaction between the geometrical urban features and the associated physical hydraulic processes. The proposed novel technique is based on a fronttracking variant of the lattice Boltzmann method that is here 


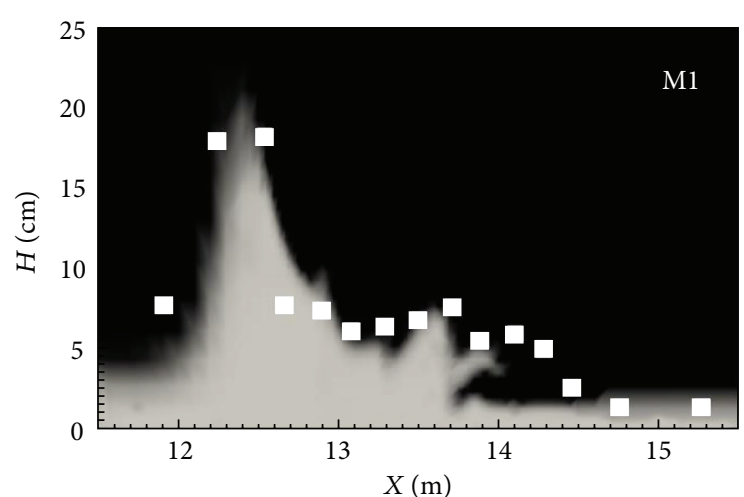

(a)

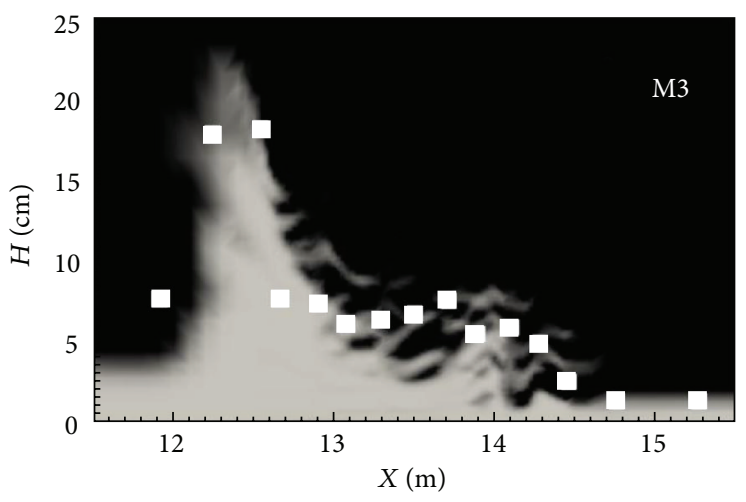

(c)

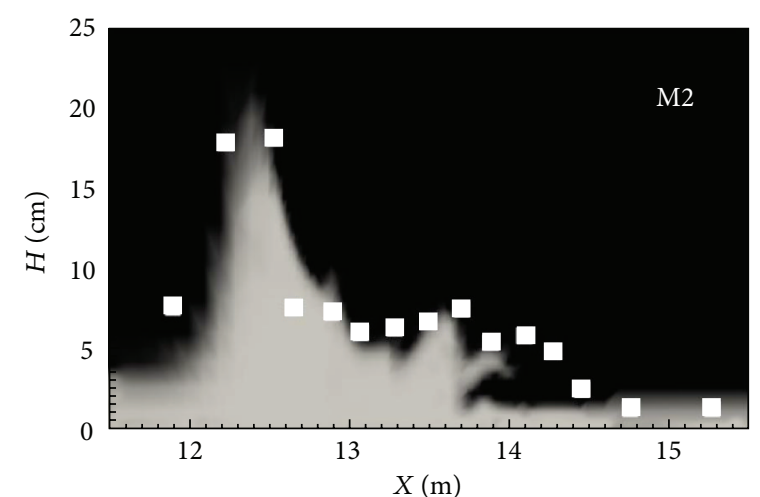

(b)

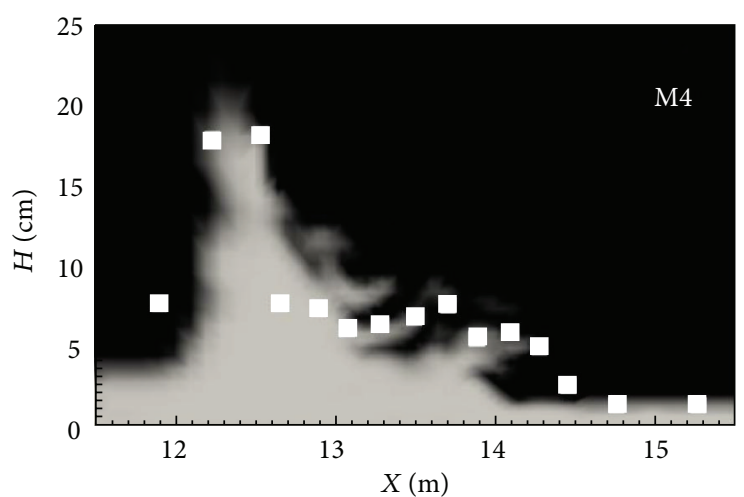

(d)

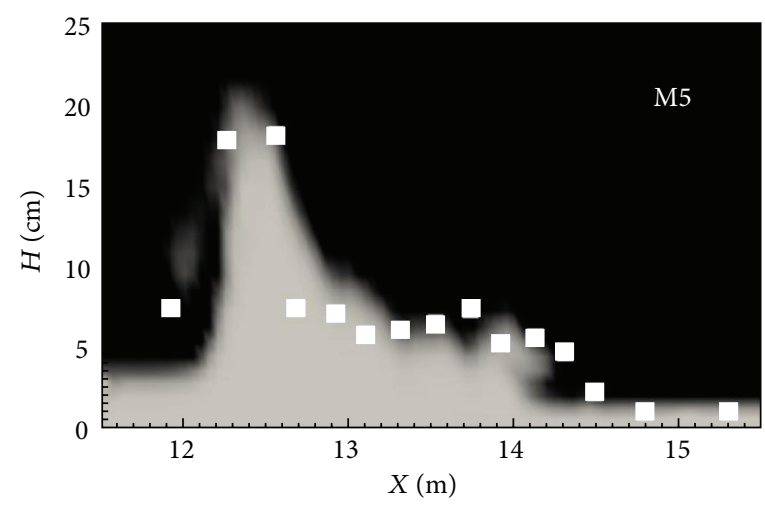

(e)

FIGURE 13: Comparison of experimental free-surface value (points) and simulated fraction fill results for 6 different computational meshes at time $=4 \mathrm{~s}$ from the release at section $Y=2 \mathrm{~m}$

investigated as a valid alternative to the standard numerical Navier-Stokes model in the treatment of instantaneously varying flows interacting with artificial structures. In particular, test cases evaluate the accuracy and the computational efficiency of numerical simulations with respect to analytical solutions and experimental data. The critical analysis of model performances provides the following conclusive remarks.

The proposed LBM is able to predict with a high level of accuracy typical hydraulic phenomena characterizing engineering applications related to the flooding of simple straight channels (test (a)) and asymmetrical front waves in rectangular basins (test (b)). More specifically, as compared to the fully 3D NS model, it is reported the validity and robustness of LBM results that, while implementing the same resolution of the geometric domain, are demonstrated to be more computationally efficient (test (b)).

The last test investigates the diverse hydraulic unsteady processes, like the hydraulic jump, return, and diverged flow, that originate from the passage of the flood wave through a synthetic urban setting (test (c)). The application of the presented 3D numerical schemes shows the major advantages 


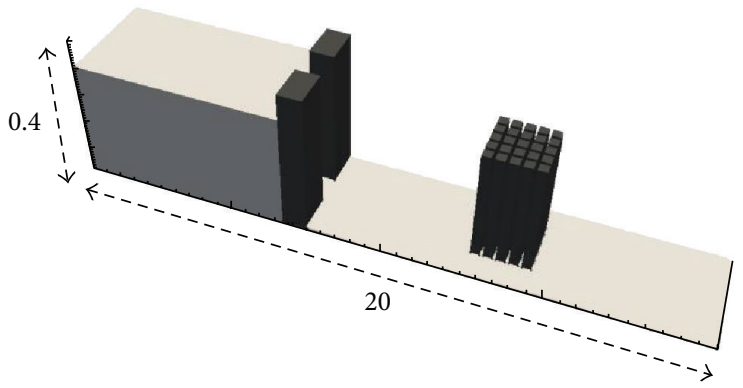

(a)

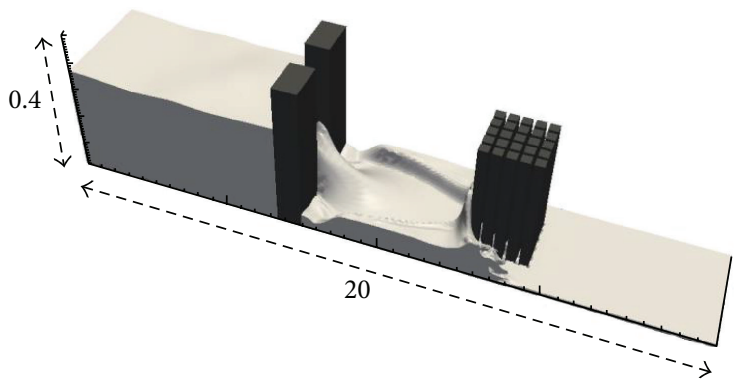

(c)

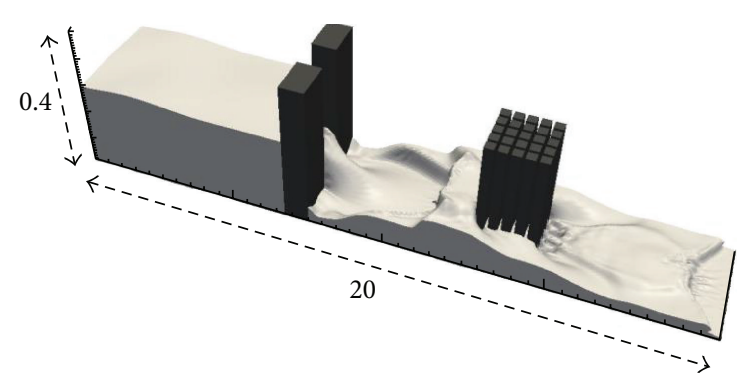

(e)

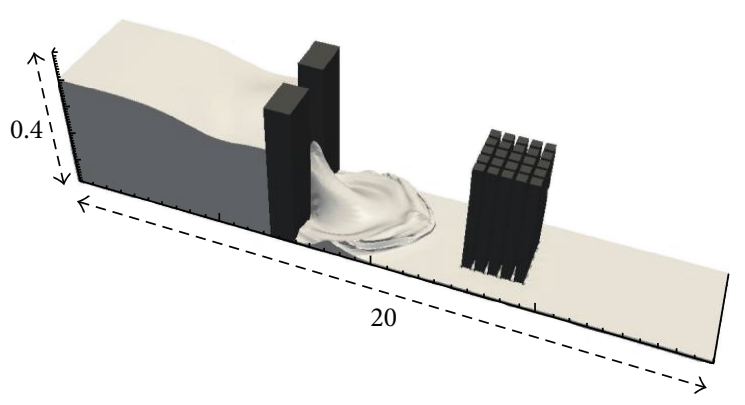

(b)

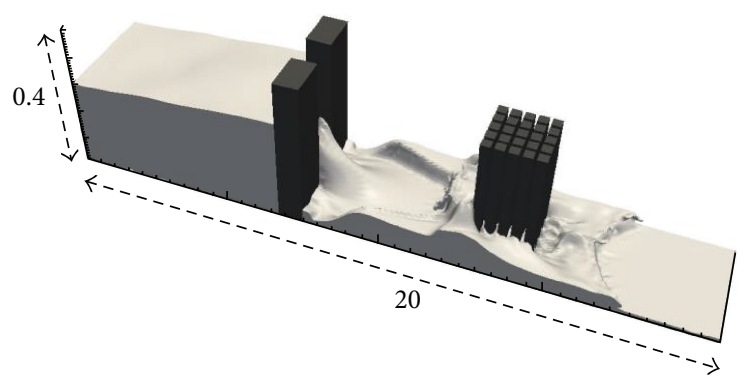

(d)

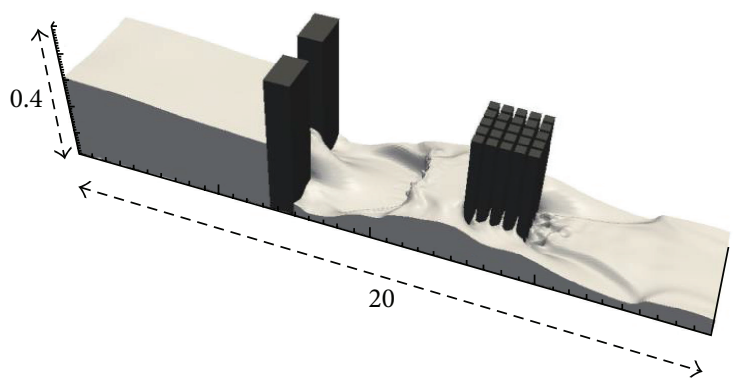

(f)

FIGURE 14: Free-surface evolution for scenario M5 for different time steps $((a)=0 \mathrm{~s},(\mathrm{~b})=2 \mathrm{~s},(\mathrm{c})=4 \mathrm{~s},(\mathrm{~d})=6 \mathrm{~s},(\mathrm{e})=8 \mathrm{~s}$, and (f) $=10 \mathrm{~s}$ ).

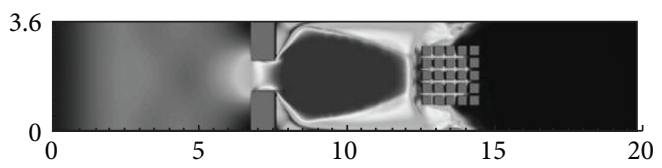

(a)

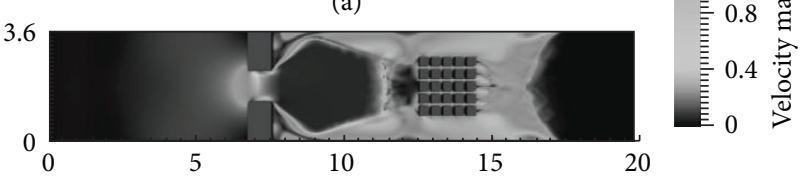

(b)

FIGURE 15: Evolution of velocity magnitude for scenario M5 at different time steps $-t=4 \mathrm{~s} \mathrm{(a)}$, and $6 \mathrm{~s} \mathrm{(b).}$

and disadvantages of both LBM and NS schemes confirming the ability of LBM in representing such abruptly changing and irregular flow dynamics. Nevertheless, the mesoscopic LBM approach is significantly bound by the regular structured mesh, while the continuous NS model performs well, also in large domains, especially when using a more accurate grid
(M5) that is adaptively refined to more accurately represent obstacles.

\section{References}

[1] P. Manciola, S. Di Francesco, and C. Biscarini, "Flood protection and risk management: the case of Tescio River basin," in Proceedings of the Role of Hydrology in Water Resources Management Symposium, vol. 327 of IAHS Red Book Series, pp. 174-183, Capri, Italy, October 2008.

[2] Y. Jia and S. S. Y. Wang, "Numerical model for channel flow and morphological change studies," Journal of Hydraulic Engineering, vol. 125, no. 9, pp. 924-933, 1999.

[3] P. G. Manciola, A. Mazzoni, and F. Savi, "Formation and propagation of steep waves: an investigative experimental interpretation," in Proceedings of the Specialty Conference on Modelling of Flood Propagation Over Initially Dry Areas, pp. 283-297, Milano, Italy, July 1994.

[4] C. Biscarini, "Computational fluid dynamics modeling of landslide generated water waves," Landslide Springer, vol. 7, no. 2, pp. 117-124, 2010. 
[5] A. Ferrari, L. Fraccarollo, M. Dumbser, E. F. Toro, and A. Armanini, "Three-dimensional flow evolution after a dam break," Journal of Fluid Mechanics, vol. 663, pp. 456-477, 2010.

[6] C. Biscarini, S. Di Francesco, and P. Manciola, "CFD modelling approach for dam break flow studies," Hydrology and Earth System Sciences, vol. 14, no. 4, pp. 705-718, 2010.

[7] D. Dutykh and D. Mitsotakis, "On the relevance of the dam break problem in the context of nonlinear shallow water equations," Discrete and Continuous Dynamical Systems B, vol. 13, no. 4, pp. 799-818, 2010.

[8] H. Liu, J. G. Zhou, and R. Burrows, "Lattice Boltzmann simulations of the transient shallow water flows," Advances in Water Resources, vol. 33, no. 4, pp. 387-396, 2010.

[9] C. L. Mader, "Modeling the 1958 Lituya Bay Mega-Tsunami, II," Science of Tsunami Hazards, vol. 20, pp. 241-250, 2002.

[10] C. Biscarini and M. Testa, "Three-dimensional numerical modelling of the marmore water falls," Progress in Computational Fluid Dynamics, vol. 11, no. 2/2011, pp. 105-115, 2011.

[11] R. Benzi, S. Succi, and M. Vergassola, "The lattice Boltzmann equation: theory and applications," Physics Report, vol. 222, no. 3, pp. 145-197, 1992.

[12] S. Succi, The Lattice Boltzmann Equation for Fluid Dynamics and Beyond, Numerical Mathematics and Scientific Computation, The Clarendon Press, Oxford, UK, 2001.

[13] S. Ubertini, P. Asinari, and S. Succi, "Three ways to lattice Boltzmann: a unified time-marching picture," Physical Review E, vol. 81, no. 1, Article ID 016311, 2010.

[14] J. F. Chau, D. Or, and M. C. Sukop, "Simulation of gaseous diffusion in partially saturated porous media under variable gravity with lattice Boltzmann methods," Water Resources Research, vol. 41, no. 8, Article ID W08410, 2005.

[15] X. Shan and H. Chen, "Lattice Boltzmann model for simulating flows with multiple phases and components," Physical Review E, vol. 47, pp. 1815-1819, 1993.

[16] D. Chiappini, G. Bella, S. Succi, F. Toschi, and S. Ubertini, "Improved lattice Boltzmann without parasitic currents for Rayleigh-Taylor instability," Communications in Computational Physics, vol. 7, no. 3, pp. 423-444, 2010.

[17] G. Falcucci, S. Ubertini, and S. Succi, "Lattice Boltzmann simulations of phase-separating flows at large density ratios: the case of doubly-attractive pseudo-potentials," Soft Matter, vol. 6, no. 18, pp. 4357-4365, 2010.

[18] Y. Peng, J. G. Zhou, and R. Burrows, "Modeling free-surface flow in rectangular shallow basins by using lattice boltzmann method," Journal of Hydraulic Engineering, vol. 137, no. 12, pp. 1680-1685, 2012.

[19] G. Falcucci, G. Bella, G. Chiatti, S. Chibbaro, M. Sbragaglia, and S. Succi, "Lattice Boltzmann models with mid-range interactions," Communications in Computational Physics, vol. 2, no. 6, pp. 1071-1084, 2007.

[20] C. Biscarini, S. Di Francesco, F. Nardi, and P. Manciola, "Threedimensional hydraulic modelling of unsteady free-surface flows using the Lattice Boltzmann Method," Journal of Hydraulic Engineering. In review.

[21] J. G. Zhou, Lattice Boltzmann Methods For Shallow Water flows, Springer, 2004.

[22] Y. Li and P. Huang, "A coupled lattice Boltzmann model for the shallow water-contamination system," International Journal for Numerical Methods in Fluids, vol. 59, pp. 195-213, 2009.

[23] C. Körner, M. Thies, T. Hofmann, N. Thürey, and U. Rüde, "Lattice Boltzmann model for free surface flow for modeling foaming," Journal of Statistical Physics, vol. 121, no. 1-2, pp. 179196, 2005.

[24] N. Thürey, T. Pohl, U. Rüde, M. Öchsner, and C. Körner, “Optimization and stabilization of LBM free surface flow simulations using adaptive parameterization," Computers and Fluids, vol. 35, no. 8-9, pp. 934-939, 2006.

[25] C. Biscarini, S. D. Francesco, and M. Mencattini, "Application of the lattice Boltzmann method for large-scale hydraulic problems," International Journal of Numerical Methods for Heat and Fluid Flow, vol. 21, no. 5, pp. 584-601, 2011.

[26] G. Falcucci, S. Ubertini, C. Biscarini et al., "Lattice boltzmann methods for multiphase flow simulations across scales," Communications in Computational Physics, vol. 9, no. 2, pp. 269-296, 2011.

[27] H. M. Solo-Gabriele and F. E. Perkins, "Streamflow and suspended sediment transport in an urban environment," Journal of Hydraulic Engineering, vol. 123, no. 9, pp. 807-811, 1997.

[28] S. Soares-Frazão and Y. Zech, "Experimental study of dambreak flow against an isolated obstacle," Journal of Hydraulic Research, vol. 45, pp. 27-36, 2007.

[29] S. Di Francesco, C. Biscarini, and P. Manciola, "Mesoscopic modeling of dam break flow," in Proceedings of the 3rd International Junior Researcher and Engineer Workshop on Hydraulic Structures (IJREWHS '10), R. Jannsenn and H. Chanson, Eds., Hydraulic Model Report CH80/10, School of Civil Eng., The University of Queensland, Brisbane, Australia, 2010.

[30] D. Yu and S. N. Lane, "Urban fluvial flood modelling using a two dimensional diffusion-wave treatment: 1 . Mesh resolution effects," Hydrological Processes, vol. 20, pp. 1541-1565, 2006.

[31] S. Zaleski, J. Li, and S. Succi, "Two-dimensional Navier-Stokes simulation of deformation and breakup of liquid patches," Physical Review Letters, vol. 75, pp. 244-247, 1995.

[32] O. Ubbink and R. I. Issa, "A method for capturing sharp fluid interfaces on arbitrary meshes," Journal of Computational Physics, vol. 153, no. 1, pp. 26-50, 1999.

[33] Open FOAM, User Guide, Version 1.5, The Open Source CFD Toolbox, 2008.

[34] P. L. Bhatnagar, E. P. Gross, and M. Krook, "A model for collision processes in gases. I. Small amplitude processes in charged and neutral one-component systems," Physical Review, vol. 94, no. 3, pp. 511-525, 1954.

[35] S. Chen and G. D. Doolen, "Lattice Boltzmann method for fluid flows," Annual Review of Fluid Mechanics, vol. 30, pp. 329-364, 1998.

[36] F. Tosi, S. Ubertini, S. Succi, H. Chen, and I. V. Karlin, "Numerical stability of entropic versus positivity-enforcing lattice Boltzmann schemes," Mathematics and Computers in Simulation, vol. 72, no. 2-6, pp. 227-231, 2006.

[37] P. A. Skordos, "Initial and boundary conditions for the lattice Boltzmann method," Physical Review E, vol. 48, no. 6, pp. 4823$4842,1993$.

[38] T. Inamuro, M. Yoshino, and F. Ogino, "A non-slip boundary condition for lattice Boltzmann method," Journal of Computational Physics, vol. 155, p. 307, 1999.

[39] B. Galperin and S. A. Orszag, Large Eddy Simulation of Complex Engineering and Geophysical Flows, Cambridge University Press, New York, NY, USA, 1993.

[40] J. Smagorinsky, "General circulation experiments with the primitive equations. I. The basic experiment," Monthly Weather Review, vol. 91, pp. 99-164, 1963. 
[41] A. Ritter, "The propagation of water waves," Ver Deutsch ingenieur zeitschr, vol. 36, no. 33, part 3, pp. 947-954, 1892.

[42] R. Dressler, "Comparison of theories and experiments for the hydraulic dam-break wave," Proceedings of the International Association of Scientific Hydrology Assemblée Générale, Rome, Italy, vol. 3, no. 38, pp. 319-328, 1954.

[43] H. Chanson, "Application of the method of characteristics to the dam break wave problem," Journal of Hydraulic Research, vol. 47, no. 1, pp. 41-49, 2009.

[44] Y. Cavaillé, Contribution à l'étude de l'écoulement variable accompagnant la vidange brusque d'une retenue, vol. 410 of Publications Scientifiques et Techniques du Ministère de l'air, Paris, France, 1965.

[45] F. Alcrudo and P. Garcia-Navarro, "High-resolution Godunovtype scheme in finite volumes for the $2 \mathrm{D}$ shallow-water equations," International Journal for Numerical Methods in Fluids, vol. 16, no. 6, pp. 489-505, 1993.

[46] R. J. Fennema and M. H. Chaudhry, "Explicit methods for 2-D transient free-surface flows," Journal of Hydraulic Engineering, vol. 116, no. 8, pp. 1013-1034, 1990.

[47] S. Soares-Frazão and Y. Zech, "Dam-break flow through an idealised city," Journal of Hydraulic Research, vol. 46, no. 5, pp. 648-658, 2008.

[48] O. Filippova and D. Hänel, "Grid Refinement for Lattice-BGK Models," Journal of Computational Physics, vol. 147, no. 1, pp. 219-228, 1998. 


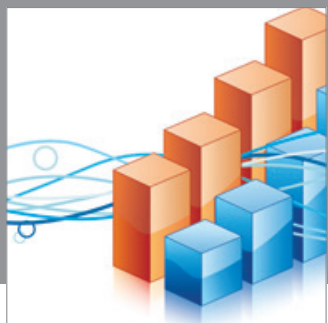

Advances in

Operations Research

mansans

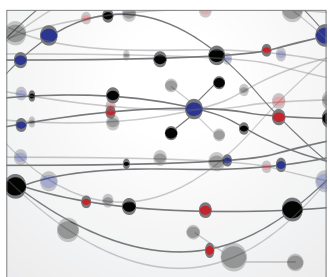

The Scientific World Journal
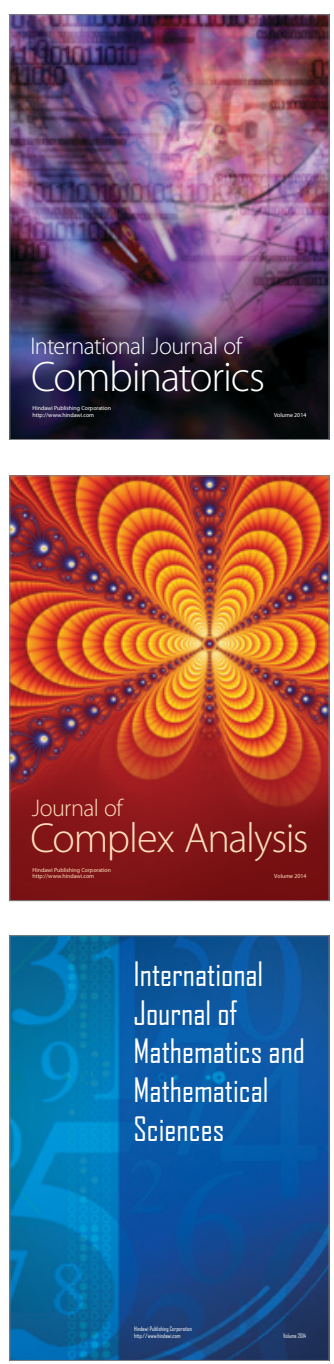
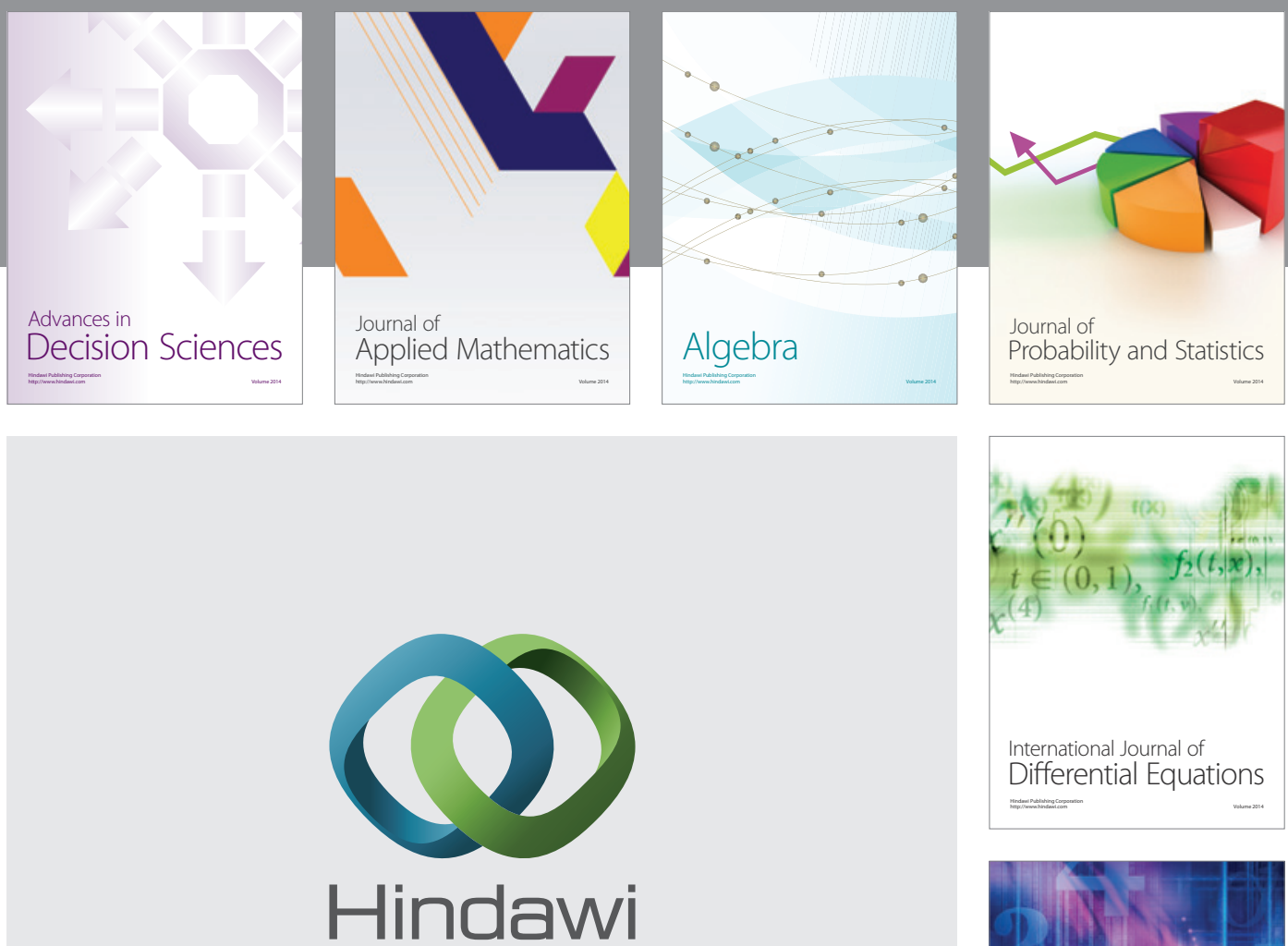

Submit your manuscripts at http://www.hindawi.com
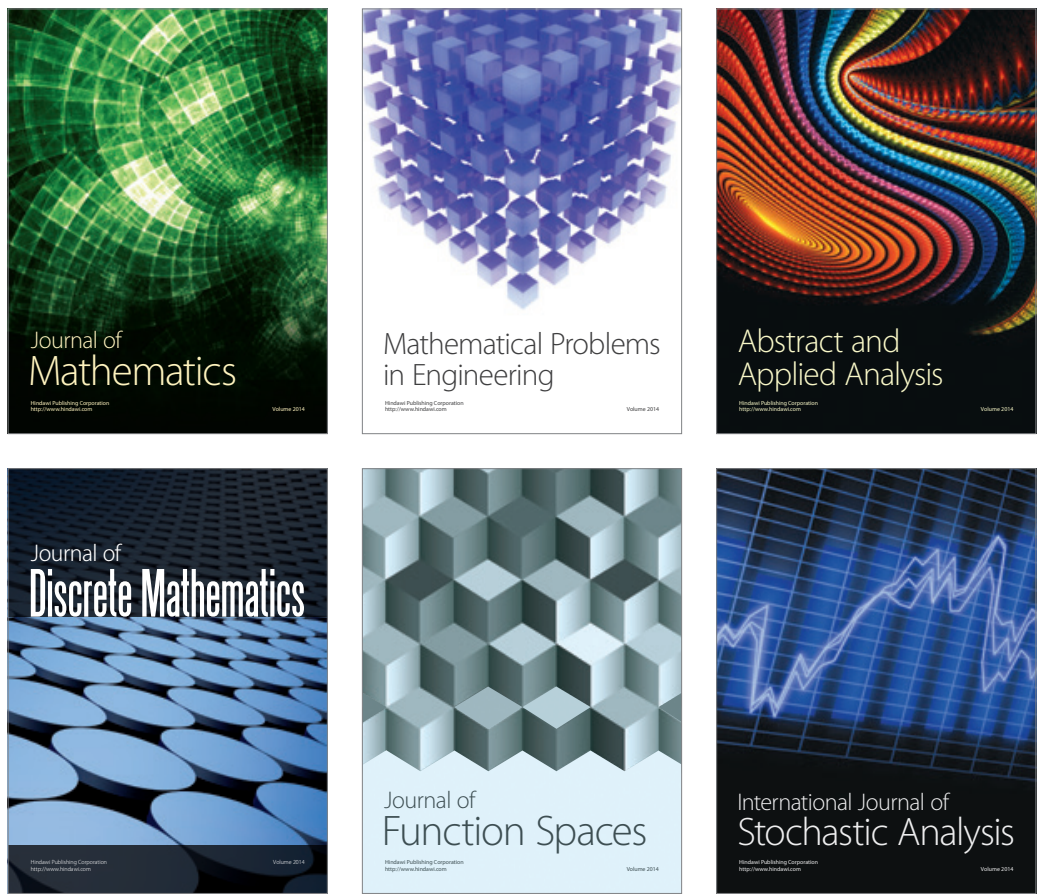

Journal of

Function Spaces

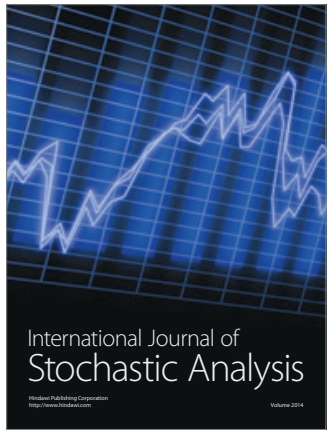

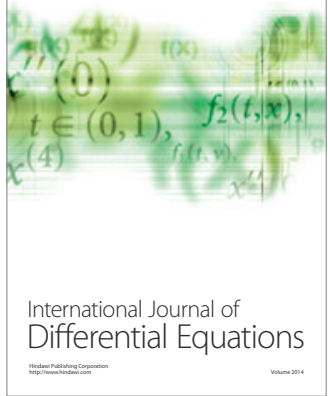
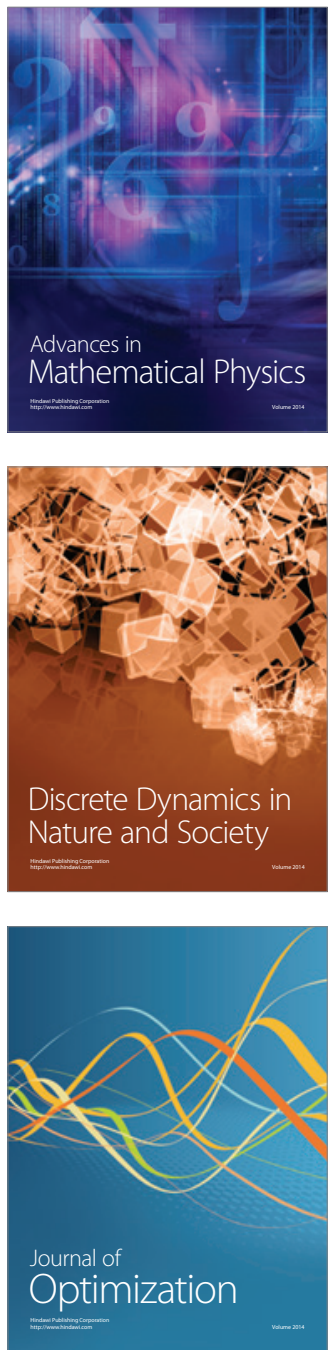